\title{
Brownian motions on the 3-dimensional rotation group
}

\author{
By
}

H. P. McKean, JR.

(Received January 4, 1960)

(Communicated by Prof. K. Itô)

\section{INTRODUCTION}

K. Itô [1950]* and K. Yosida [1952] defined and constructed all the Brownian motions on Lie groups. The purpose of the present paper is to give a new method for constructing the Brownian sample paths on the 3-dimensional rotation group $\mathrm{SO}$ (3). The idea is to inject the differentials $z(d t)$ of a (skew) Brownian motion on the Lie algebra into $\mathrm{SO}(3)$ via the exponential map $e$ and to piece the resulting infinitessimal rotations $e[z(d t)]$ into a continuous path (product integral):

1.1

$$
\begin{aligned}
& \mathrm{g}_{\infty}(t) \\
& =\bigcap_{s \leq t} e[z(d s)] \\
& =\lim _{n \uparrow \infty} e\left[z\left(0,2^{-n}\right)\right] \cdots e\left[z\left(2^{-n}\left[2^{n} t\right], t\right)\right] \quad t \geq 0 .
\end{aligned}
$$

The same trick gives the Brownian motions on all the classical (non-exceptional) simple Lie groups of É. Cartan's list.

F. Perrin [1928] computed the counterparts of the Gauss and Poisson laws on SO (3); for a sketch of Perrin's results, see P. Lévy [1948: 194-203].

I divide the paper into 8 sections: 2 deals with $\mathrm{SO}(3)$, its Lie algebra, and its differential operators; 3 with its Brownian motions. 4 states the program of injection. 5 is devoted to sums $\mathfrak{g}=\sum_{n \geq 0} \dot{i}_{n}$ of stochastic integrals

* K. Itô [1950] means K. Itô's 1950 publication listed at the end of this paper; K. Itô $[1950: 6-8]$ would mean pages $6-8$ of that work. 
1. $2 \quad \dot{\mathrm{j}}_{0}=\mathrm{e}, \quad \dot{\mathrm{i}}_{n}=\int_{0}^{t} \dot{\mathrm{j}}_{n-1} \mathrm{i}(d s) \quad \mathrm{j}(d s)=\mathfrak{z}(d s)+\frac{1}{2} z(d s)^{2}$.

6 contains the identification of $\mathfrak{g}$ with $\mathrm{g}_{\infty}=\bigcap_{s \leq t} e[z(d s)]$. 7 identifies $\mathrm{g}=\mathrm{g}_{\infty}$ with a Brownian motion on $\mathrm{SO}(3) .8$ contains an example which C. D. Gorman [1958] has also treated*. I will suppose, for the purposes of 5,6 , and 7 , that the reader is familiar with stochastic integrals as presented, for example, in K. Itô [1951].

I wish to thank $\mathrm{H}$. Trotter who suggested the problem of 8 which was the starting point of this paper. I must also thank K. Itô for helpful talks and for a trick used in 5 .

\section{ROTATION GROUP}

$R^{3}$ is the (real) 3 -dimensional euclidean space of points $x=\left(x_{1}, x_{2}, x_{3}\right)$, etc. $;|x|=\sqrt{ } x_{1}^{2}+x_{2}^{2}+x_{3}^{2} ; e_{1}=(1,0,0), e_{2}=(0,1,0)$, $e_{3}=(0,0,1) ; \times$ is the outer product for $R^{3}\left(e_{1} \times e_{2}=e_{3}, e_{2} \times e_{3}=e_{1}\right.$, $e_{3} \times e_{1}=e_{2}$ ); small German letters f, etc. stand for (real) $3 \times 3$ matrices; $* f$ is the transpose of $f ; f^{-1}$ its inverse; $|f|$ its norm; $\mathrm{SO}(3)$ is the (multiplicative) group of $3 \times 3$ orthogonal matrices $\mathfrak{g}\left(\mathfrak{g}^{*} \mathfrak{g}=\mathfrak{e}=\right.$ the unit $)$ of determinant +1 .

Bringing in the infinitessimal rotations

$2.1 \quad \mathfrak{e}_{1}=\left(\begin{array}{rrr}0 & 0 & 0 \\ 0 & 0 & -1 \\ 0 & 1 & 0\end{array}\right), \quad \mathfrak{e}_{2}=\left(\begin{array}{rrr}0 & 0 & 1 \\ 0 & 0 & 0 \\ -1 & 0 & 0\end{array}\right), \quad \mathfrak{e}_{3}=\left(\begin{array}{rrr}0 & -1 & 0 \\ 1 & 0 & 0 \\ 0 & 0 & 0\end{array}\right)$

and the product

2.2

$$
\left[f_{1}, f_{2}\right]=f_{1} f_{2}-f_{2} f_{1},
$$

a short computation justifies

2.3

$$
\left[\mathfrak{e}_{1}, \mathfrak{e}_{2}\right]=\mathfrak{e}_{3}, \quad\left[\mathfrak{e}_{2}, \mathfrak{e}_{3}\right]=\mathfrak{e}_{1}, \quad\left[\mathfrak{e}_{3}, \mathfrak{e}_{1}\right]=\mathfrak{e}_{2},
$$

which shows that the vector space $A$ of matrices

2.4

$$
\mathfrak{a}=\alpha_{1} \mathfrak{e}_{1}+\alpha_{2} \mathfrak{e}_{2}+\alpha_{3} \mathrm{e}_{3} \quad \alpha=\left(\alpha_{1}, \alpha_{2}, \alpha_{3}\right) \in R^{3}
$$

under the product 2.2 is isomorphic to $R^{3}$ under the outer product. $A$ is the so-called Lie algebra of $\mathrm{SO}(3)$.

$A$ is connected to $\mathrm{SO}(3)$ via the exponential map 2.5

$$
e(\mathfrak{f})=\sum_{n \geq 0} \mathfrak{f}^{n} / n !
$$

* Note added in proof : for a complete account, see C. D. Gorman, Brownian motion of rotation. Trans. Amer. Math. Soc. 94 (1960), 103-117. 
and the logarithm

$$
l(\mathfrak{f})=\sum_{n \geq 1}(\mathfrak{f}-\mathrm{e})^{n} / n \quad|\mathfrak{f}-\mathfrak{e}|<1:
$$

in fact, $l$ maps the neighborhood $|\mathfrak{g}-\mathfrak{e}|<1$ of $\mathrm{SO}$ (3) onto a neighborhood of the 0 element of $A$ and $e$ maps the neighborhood $|\mathfrak{a}|<\lg 2$ of $A$ onto a neighborhood of $e$ in $\mathrm{SO}(3)$; both maps are $1: 1$; in the first case, the inverse map is $\mathfrak{e}$; in the second, it is $l$.

SO (3) is homeomorphic to the 3-dimensional projective space $P^{3}$ : in fact, $P^{3}$, viewed as the spherical surface $S^{3} \subset R^{4}$ with antipodal identifications, is homeomorphic to the solid 3-dimensional ball of diameter $2 \pi$ with antipodal surface points identified, and the map taking $\alpha(|\alpha| \leq \pi)$ into the rotation $\mathrm{g}$ of total angle $|\alpha|$ about the axis $\alpha$ in the sense of the right-hand screw rule is a homeomorphism of the solid ball with surface identifications onto SO (3) (for small $\alpha, \alpha \rightarrow g$ is just the exponential map).

Consider the class $C^{2}[\mathrm{SO}(3)]$ of functions $f=f(\mathrm{~g})$ defined on $\mathrm{SO}$ (3) such that, for $\mathfrak{g} \in \mathrm{SO}(3), h(\alpha)=f\left(\mathfrak{g} e\left[\alpha_{1} \mathrm{e}_{1}+\alpha_{2} \mathrm{e}_{2}+\alpha_{3} \mathrm{e}_{3}\right]\right)$ is of class $C_{2}$ near $\alpha=0$ and define

$$
2.7 \quad\left(\mathfrak{\Im}_{1} f\right)(\mathfrak{g})=h_{1}(0), \quad\left(\mathfrak{\xi}_{2} f\right)(\mathfrak{g})=h_{2}(0), \quad\left(\mathfrak{\xi}_{3} f\right)(\mathfrak{g})=h_{3}(0),
$$

where the subscripts stand for partials.

Writing out the power series for $f$ at $\mathfrak{g}=\mathfrak{e}$ up to terms of degree 2 , it develops that, with the commutator product 2.2,

$$
\left[\mathfrak{F}_{1}, \mathfrak{F}_{2}\right]=\mathfrak{F}_{3}, \quad\left[\mathfrak{F}_{2}, \mathfrak{F}_{3}\right]=\mathfrak{F}_{1}, \quad\left[\mathfrak{F}_{3}, \mathfrak{F}_{1}\right]=\mathfrak{F}_{2},
$$

so that the algebra of differential operators

$$
\mathfrak{\xi}=\alpha_{1} \mathfrak{\xi}_{1}+\alpha_{2} \mathfrak{\Im}_{2}+\alpha_{3} \mathfrak{\xi}_{3} \quad \alpha=\left(\alpha_{1}, \alpha_{2}, \alpha_{3}\right) \in R^{3}
$$

under the commutator product is isomorphic to the Lie algebra; under the usual product, $\mathfrak{F}_{1}, \mathfrak{F}_{2}, \mathfrak{F}_{3}$ generate the algebra of differential operators on $\mathrm{SO}(3)$ commuting with left translations.

Contracting $\mathfrak{\xi}_{1}, \mathfrak{\xi}_{2}, \mathfrak{\xi}_{3}$ to the class of functions $f \in C^{2}[\mathrm{SO}(3)]$ such that $f\left(\mathfrak{g}_{1}\right)=f\left(\mathfrak{g}_{2}\right)$ if $\mathfrak{g}_{1} e_{3}=\mathfrak{g}_{2} e_{3}\left(e_{3}=(0,0,1)\right)$ and viewing them as differential operators on the coset space $\mathrm{SO}(3) / \mathrm{SO}(2)=\mathrm{S}^{2}$ of points $g e_{3}$, one gets the following 3 operators:

$2.10 \sin \phi \frac{\partial}{\partial \psi}-\frac{\cos \psi \cos \phi}{\sin \psi} \frac{\partial}{\partial \phi}, \quad \cos \phi \frac{\partial}{\partial \psi}-\frac{\cos \psi \sin \phi}{\sin \psi} \frac{\partial}{\partial \phi}, \quad \frac{\partial}{\partial \phi}$, 
where $0 \leq \psi \leq \pi$ is the colatitude and $0 \leq \phi<2 \pi$ the longitude of $\mathfrak{g} e_{3}$.

The reader is referred to W. Maak [1950: 161-179, 209-210] and to I. Gelfand and Z. Ya. Šapiro [1956; 207-245] for proofs and more information.

\section{BROWNIAN MOTIONS ON SO (3).}

Consider the space of all continuous (sample) paths $w$ : $t \in[0,+\infty) \rightarrow \mathrm{g}(t) \in \mathrm{SO}(3)$, let $\boldsymbol{B}_{t}$ be the smallest Borel algebra of subsets of the path space measuring the entries of $g(s)$ for each $s \leq t$, let $\boldsymbol{B}$ be the smallest Borel algebra containing all of these, and take non-negative Borel measures $P_{\mathfrak{g}}$ defined on $\boldsymbol{B}$ of total mass +1 , one to each $g \in \mathrm{SO}(3)$, such that $P_{\mathrm{g}}[\mathrm{g}(0)=\mathfrak{g}]=1$ for each $\mathrm{g} \in \mathrm{SO}(3)$ and $P_{\mathrm{g}}(B)$ is Borel on SO (3) for each $B \in \boldsymbol{B}$.

$\left[W, \boldsymbol{B}, P_{\mathrm{g}}: \mathrm{g} \in \mathrm{SO}(3)\right]$ is said to be a (left) Brownian motion on $\mathrm{SO}(3)$ if it is Markov:

$$
P \cdot\left[\mathfrak{g}(t+s) \in d \mathfrak{g} \mid \boldsymbol{B}_{s}\right]=\left.P_{\mathfrak{h}}[\mathfrak{g}(t) \in d \mathfrak{g}]\right|_{\mathfrak{h}=\mathfrak{g}(s)} \quad t, s \geq 0
$$

and if it is also (left) group-invariant:

$$
P_{\mathfrak{g}}(B)=P_{\mathfrak{e}}\left(\mathfrak{g}^{-1} B\right) \quad \mathfrak{g} \in \mathrm{SO}(3), B \in \boldsymbol{B},
$$

where $\mathrm{g}^{-1} B$ is the set of sample paths such that the translated path $\mathrm{g}^{-1} \mathrm{~g}(t): t \geq 0$ lies in $B$.

Given such a Brownian motion, its generator (S) is defined as

3.3

$$
\begin{aligned}
(\mathbb{S} f)(\mathfrak{g})=\lim _{t \downarrow 0} t^{-1} E_{\mathfrak{g}}[ & f(\mathfrak{g}(t))-f(\mathfrak{g})] \\
\mathfrak{g} & \in \operatorname{SO}(3), E .(f)=\int f P .(d w)
\end{aligned}
$$

for the class $C((3)$ of functions $f \in C[\mathrm{SO}(3)]$ such that the limit exists (pointwise) and the resulting (Sf $f$ lies again in $C[\mathrm{SO}(3)]$.

Because $C(\mathbb{S})$ is a (left) ideal in $C$ under the convolution

3.4

$$
\left(f_{1} \otimes f_{2}\right)(\mathfrak{h})=\int f_{1}\left(\mathfrak{h} \mathfrak{g}^{-1}\right) f_{2}(\mathfrak{g}) d \mathfrak{g},
$$

where $d \mathfrak{g}$ is Haar measure for $\mathrm{SO}(3)$, it is clear from the inclusion $C^{2} \otimes C(\mathbb{S}) \subset C^{2}$ that $C(\mathbb{S}) \cap C^{2}$ is well-populated, and it is possible to conclude that $(S)$ is an elliptic differental operator of degree 2 commuting with (left) translations: 


$$
\mathbb{B}=\frac{1}{2} \sum_{i, j \leq 3} l_{i, j} \xi_{i} \varpi_{j}+\sum_{i \leq 3} m_{i} \mathbb{\Im}_{i},
$$

where

3. 6

$$
\mathfrak{l}=\left(\begin{array}{lll}
l_{11} & l_{12} & l_{13} \\
l_{21} & l_{22} & l_{23} \\
l_{31} & l_{32} & l_{33}
\end{array}\right)=\lim _{t \downarrow 0} t^{-1} E_{\mathfrak{c}}[\mathrm{g}(t)-\mathfrak{e}]^{2}
$$

is symmetric $(\mathfrak{l}=* \mathfrak{l})$ and non-negative definite $(\mathfrak{l} \geq 0)$, and

$$
\mathrm{m}=m_{1} \mathrm{e}_{1}+m_{2} \mathrm{e}_{2}+m_{3} \mathrm{e}_{2}=\lim _{t \downarrow 0} t^{-1} E_{\mathfrak{e}}[\mathfrak{g}(t)-\mathfrak{e}] .
$$

On the other hand, an elliptic differential operator such as 3.5 with $\mathfrak{l}=* \mathfrak{l} \geq 0$ generates a (left) Brownian motion; for the proofs, the reader is referred to the articles of K. Itô [1950] and K. Yosida [1952].

\section{INJECTION}

Consider, now, the standard Brownian motion on $R^{3}$ with generator $\frac{1}{2}\left(\frac{\partial^{2}}{\partial x_{1}^{2}}+\frac{\partial^{2}}{\partial x_{2}^{2}}+\frac{\partial^{2}}{\partial x_{3}^{2}}\right)$ and sample paths $w: t \rightarrow x(t) \in R^{3}$, write $P$ for the Wiener measure for paths starting at 0 , let $E(f)=\int f P(d w)$, select an $\mathrm{SO}(3)$ Brownian generator (\$s as in 3.5 , introduce the (skew) $R^{3}$ Brownian motion

4.1

$$
z(t)=\mathfrak{l}^{1 / 2} x(t)+m t \quad t \geq 0,
$$

where $\mathfrak{I}^{1 / 2}$ is the non-negative definite root of $\mathfrak{I}$ and $m=\left(m_{1}, m_{2}, m_{3}\right)$, and, making the identification of $R^{3}$ and $A\left(e_{1} \rightarrow \mathfrak{e}_{1}, e_{2} \rightarrow \mathfrak{e}_{2}, e_{3} \rightarrow \mathfrak{e}_{3}\right)$, think of 4.1 as a Brownian motion $z=z_{1} \mathfrak{e}_{1}+z_{2} \mathfrak{e}_{2}+z_{3} \mathfrak{e}_{3}$ in the Lie algebra itself.

We inject $z$ into SO (3) via the exponental, thus:

$$
\begin{array}{ccc}
\mathrm{g}_{n}(t)=\mathrm{g}(0) \in \mathrm{SO}(3) & t=0 \\
=\mathrm{g}_{n}\left(j 2^{-n}\right) e[z(\Delta)] & t>0 \\
\Delta=\left[j 2^{-n}, t\right), \quad j=\left[2^{n} t\right], \quad z(\Delta)=z(t)-z\left(j 2^{-n}\right)
\end{array}
$$

and assert that $\mathrm{g}_{n}$ converges to a limit $\mathfrak{g}_{\infty}$, that the convergence is uniform on compacts, and that $\mathrm{g}_{\infty}$ is the Brownian motion on SO (3) with generator $(S)$.

With the help of P. Lévy's Hölder condition [1937 : 168-172] 
4.3

$$
\lim _{\substack{t=t_{2}-t_{1} \downarrow 0 \\ 0 \leq t_{1} \leq t_{2} \leq 1}} \frac{\left|x\left(t_{2}\right)-x\left(t_{1}\right)\right|}{\sqrt{ } 2 t|\lg t|}=1
$$

and the multiplication table

$$
\begin{aligned}
& 4.4 e_{1}^{2}=\left(\begin{array}{rrr}
0 & 0 & 0 \\
0 & -1 & 0 \\
0 & 0 & -1
\end{array}\right), \quad e_{2}^{2}=\left(\begin{array}{rrr}
-1 & 0 & 0 \\
0 & 0 & 0 \\
0 & 0 & -1
\end{array}\right), \quad e_{3}^{2}=\left(\begin{array}{rrr}
-1 & 0 & 0 \\
0 & -1 & 0 \\
0 & 0 & 0
\end{array}\right) \\
& e_{1} e_{2}=\left(\begin{array}{lll}
0 & 0 & 0 \\
1 & 0 & 0 \\
0 & 0 & 0
\end{array}\right), \quad e_{2} e_{3}=\left(\begin{array}{lll}
0 & 0 & 0 \\
0 & 0 & 0 \\
0 & 1 & 0
\end{array}\right), e_{3} e_{1}=\left(\begin{array}{lll}
0 & 0 & 0 \\
0 & 0 & 0 \\
1 & 0 & 0
\end{array}\right),
\end{aligned}
$$

it is clear that, up to terms involving $z(\Delta)^{3}$ (of magnitude $<2^{-4 n / 3}$ ), $4.5 \mathrm{~g}_{n}(t)-\mathrm{g}_{n}\left(j 2^{-n}\right)=\mathrm{g}_{n}\left(j 2^{-n}\right)(e[z(\Delta)]-\mathfrak{e})=\mathrm{g}_{n}\left(j 2^{-n}\right)\left(\mathfrak{z}(\Delta)+\frac{1}{2} z(\Delta)^{2}\right)$, where

$4.6 \quad z(\Delta)=z_{1}(\Delta) e_{1}+z_{2}(\Delta) e_{2}+z_{3}(\Delta) e_{3}=\left(\begin{array}{ccc}0 & -z_{3}(\Delta) & z_{2}(\Delta) \\ z_{3}(\Delta) & 0 & -z_{1}(\Delta) \\ -z_{2}(\Delta) & z_{1}(\Delta) & 0\end{array}\right)$ and

$4.7 \quad \frac{1}{2} z(\Delta)^{2}$

$$
=\frac{1}{2}\left(\begin{array}{ccc}
-\left[z_{2}(\Delta)^{2}+z_{3}(\Delta)^{2}\right] & z_{1}(\Delta) z_{2}(\Delta) & z_{1}(\Delta) z_{3}(\Delta) \\
z_{2}(\Delta) z_{1}(\Delta) & -\left[z_{3}(\Delta)^{2}+z_{1}(\Delta)^{2}\right] & z_{2}(\Delta) z_{3}(\Delta) \\
z_{3}(\Delta) z_{1}(\Delta) & z_{3}(\Delta) z_{2}(\Delta) & -\left[z_{1}(\Delta)^{2}+z_{2}(\Delta)^{2}\right]
\end{array}\right),
$$

and the reader who knows stochastic integrals will at once conjecture that $\mathfrak{g}_{\infty}=\lim _{n \uparrow \infty} \mathfrak{g}_{n}$ is the solution of

4.8

$$
\mathfrak{g}(t)=\mathfrak{g}(0)+\int_{0}^{t} \mathfrak{g}(s) \mathrm{j}(d s) \quad t \geq 0,
$$

where

4.9

$$
\begin{gathered}
\mathfrak{j}(d s)=\mathfrak{\jmath}(d s)+\frac{1}{2} \mathfrak{f} d s \\
\mathfrak{f}=\frac{1}{2}\left(\begin{array}{ccc}
-\left[l_{22}+l_{33}\right] & l_{12} & l_{13} \\
l_{21} & -\left[l_{33}+l_{11}\right] & l_{23} \\
l_{31} & l_{32} & -\left[l_{11}+l_{22}\right]
\end{array}\right) ;
\end{gathered}
$$

$\mathfrak{f}$ is computed from 4.7 using the multiplication table 
4. 10

\begin{tabular}{|c|c|c|c|c|}
\hline \multicolumn{1}{|c|}{} & $d t$ & $x_{1}(d t)$ & $x_{2}(d t)$ & $x_{3}(d t)$ \\
\hline$d t$ & 0 & 0 & 0 & 0 \\
\hline$x_{1}(d t)$ & 0 & $d t$ & 0 & 0 \\
\hline$x_{2}(d t)$ & 0 & 0 & $d t$ & 0 \\
\hline$x_{3}(d s)$ & 0 & 0 & 0 & $d t$ \\
\hline
\end{tabular}

and the resulting

4. 11

\begin{tabular}{|c|c|c|c|c|}
\hline$d t$ & $z_{1}(d t)$ & $z_{2}(d t)$ & $z_{3}(d t)$ \\
\hline$d t$ & 0 & 0 & 0 & 0 \\
\hline$z_{1}(d t)$ & 0 & $l_{11} d t$ & $l_{12} d t$ & $l_{13} d t$ \\
\hline$z_{2}(d t)$ & 0 & $l_{21} d t$ & $l_{22} d t$ & $l_{23} d t$ \\
\hline$z_{3}(d t)$ & 0 & $l_{31} d t$ & $l_{32} d t$ & $l_{33} d t$ \\
\hline
\end{tabular}

and $\int \mathfrak{g} d \mathfrak{j}$ means perform the matrix multiplication $\mathfrak{g} d \mathfrak{j}$ and compute the 9 resulting stochastic integrals.

The program is to solve 4.8 for $\mathfrak{g}$ and to compare $\mathfrak{g}$ and $\mathfrak{g}_{n}$ : the result will be that $\lim _{n \uparrow \infty} \mathfrak{g}_{n}=\mathfrak{g}$, permitting the identification of the product integral $\bigcap_{s \leq t} e[z(d s)]$ with $\mathfrak{g}$ and, at the same time, proving its existence.

\section{SOLVING THE INTEGRAL EQUATION}

Given (constant) $\mathfrak{g}(0) \in \mathrm{SO}(3)$, consider the continuous $\mathrm{SO}(3)$ solutions $\mathfrak{g}=\mathrm{g}(t)$ of 4.8 such that, for each $t \geq 0, \mathfrak{g}(t)$ depends upon $z(s): s \leq t$ alone.

Given 2 such solutions $g_{1}$ and $g_{2}$, their difference $\mathfrak{g}=\mathfrak{g}_{2}-\mathfrak{g}_{1}$ satisfies

5. 1

$$
\mathrm{g}(t)=\int_{0}^{t} \mathfrak{g}(s) \mathrm{j}(d s) \quad t \geq 0
$$

and, using a formula of K. Itô [1951: 60], it develops that 
5.2

$$
\begin{aligned}
\mathrm{g}(t) & * \mathrm{~g}(t) \\
& =\int_{0}^{t} \mathfrak{g} d \mathrm{j}^{*} \mathrm{~g}+\int_{0}^{t} \mathrm{~g} * d \mathrm{j} * \mathfrak{g}+\int_{0}^{t} \mathfrak{g} d \mathrm{j}^{*} d \mathrm{j}^{*} \mathfrak{g} \\
& =\int_{0}^{t} g(d \mathrm{j}+* d \mathrm{j}+d \mathrm{j} * d \mathrm{j}) * \mathfrak{g} .
\end{aligned}
$$

But, according to the multiplication table 4.11,

$$
d \dot{\mathrm{j}}+* d \mathfrak{\mathrm { j }}=\mathfrak{\mathfrak { l }} d s, \quad d \mathfrak{\mathrm { j }} * d \mathfrak{\mathrm { j }}=d \mathfrak{z} * d \mathfrak{z}=-\mathfrak{l} d s,
$$

and therefore $\mathfrak{g}^{*} \mathfrak{g}=0$, which is impossible unless $\mathfrak{g}_{2}=\mathfrak{g}_{1}$.

Consider, next, the presumptive solution

5.4

$$
\begin{gathered}
\mathrm{g}=\sum_{n \geq 0} \dot{\mathrm{I}}_{n} \\
\dot{\mathrm{I}}_{n}(t)=\int_{0}^{t} \dot{\mathrm{I}}_{n-1} d \mathrm{j} \quad n \geq 1, \quad \dot{\mathrm{I}}_{0}=\mathrm{g}(0)
\end{gathered}
$$

and let us check that the sum is convergent using the following trick of K. Itô.

Given $f$, if $\gamma_{1}\left(=|\mathfrak{f}|^{2}\right) \geq \gamma_{2} \geq \gamma_{3}$ are the eigenvalues of $f * f$, if $\mathfrak{p}_{1}$, $\mathfrak{p}_{2}, \mathfrak{p}_{3}$ are the corresponding projections $\left(\gamma_{1} \mathfrak{p}_{1}+\gamma_{2} \mathfrak{p}_{2}+\gamma_{3} \mathfrak{p}_{3}=f * f\right)$, and if $\int_{S^{2}} d o$ is the arithmetical average over the spherical surface $S^{2}$, then

5.5

$$
\int_{S^{2}}\left|\mathfrak{p}_{1} o\right|^{2} d o=\int_{S^{2}}\left|\mathfrak{p}_{2} O\right|^{2} d o=\int_{S^{2}}\left|\mathfrak{p}_{3} O\right|^{2} d o=\frac{1}{3}
$$

and

5. 6

$$
\int_{S^{2}} o f * f o d o \geq \gamma_{1} \int_{S^{2}}\left|\mathfrak{p}_{1} o\right|^{2} d o=\frac{1}{3}|f|^{2},
$$

where $o f^{*} f o$ is the inner product of $o \in S^{2}$ and $f * f o \in R^{3}$.

Viewing $\mathfrak{I}^{1 / 2} x(t): t \geq 0$ as a Brownian motion $\mathfrak{y}$ in the Lie algebra,

$$
\dot{\mathrm{j}}_{n}(t)=\int_{0}^{t} \dot{\mathrm{i}}_{n-1} \mathfrak{i} d s+\int_{0}^{t} \dot{\mathrm{I}}_{n-1} \mathfrak{l}(d s) \quad \mathfrak{i}=\frac{1}{2} \mathfrak{l}+\mathrm{m},
$$

and using 5.6 to check

$$
\begin{aligned}
5.8 \quad E & \left|\int_{0}^{t} \dot{\mathrm{I}}_{n-1} \mathfrak{y}(d s)\right|^{2} \\
& \leq 3 E \int_{s^{2}} o\left[\int_{0}^{t} \dot{\mathrm{I}}_{n-1} \mathfrak{y}(d s)^{*} \int_{0}^{t} \dot{\mathrm{I}}_{n-1} \mathfrak{y}(d s)\right] o d o \\
& =3 E \int_{s^{2}} o\left[\int_{0}^{t} \dot{\mathrm{I}}_{n-1} \mathfrak{y}(d s)^{*} \mathfrak{y}(d s)^{*} \dot{\mathrm{j}}_{n-1}\right] o d o \\
& =-3 E \int_{s^{2}} o\left[\int_{0}^{t} \dot{\mathrm{I}}_{n-1} \mathfrak{l}^{*} \dot{\mathrm{I}}_{n-1} d s\right] o d o \leq 3|\mathfrak{l}| E \int_{0}^{t}\left|\dot{\mathrm{I}}_{n-1}\right| d s,
\end{aligned}
$$


it is seen that

5.9

$$
\begin{aligned}
\left(E\left|\dot{\mathrm{j}}_{n}(t)\right|^{2}\right)^{1 / 2} & \\
& \leq\left(E\left|\int_{0}^{t} \dot{\mathrm{j}}_{n-1} \dot{\mathfrak{t}} d s\right|^{2}\right)^{1 / 2}+\left(E\left|\int_{0}^{t} \dot{\mathrm{I}}_{n-1} \mathfrak{b}(d s)\right|^{2}\right)^{1 / 2} \\
& \leq\left(|\mathfrak{i}| t^{1 / 2}+(3|\mathfrak{t}|)^{1 / 2}\right)\left(E \int_{0}^{t}\left|\dot{\mathrm{f}}_{n-1}\right|^{2} d s\right)^{1 / 2} .
\end{aligned}
$$

As to $\sum_{n \geq 0} \dot{\mathfrak{l}}_{n}$, since $\mathfrak{h}_{n}=\dot{\mathrm{j}}_{n}-E\left(\dot{\mathrm{j}}_{n}\right)$ is a martingale, $\left|\mathfrak{h}_{n}\right|$ is a semimartingale, so that, using a result of Doob [1953: 314-315] in conjunction with 5.9 ,

5. 10

$$
\begin{aligned}
P\left(\max _{s \leq t}\left|\mathfrak{h}_{n}(s)\right| \geq 2^{-n}\right) \\
\quad \leq 2^{2 n} E\left|\mathfrak{h}_{n}\right|^{2} \\
\quad \leq 32^{2 n} E \int_{S^{2}} o \mathfrak{h}_{n} * \mathfrak{h}_{n} o d o \\
\quad \leq 32^{2 n} E \int_{s^{2}} o\left[\dot{\mathfrak{i}}_{n} * \dot{\mathfrak{l}}_{n}-E\left(\dot{\mathfrak{j}}_{n}\right) * E\left(\dot{\mathfrak{l}}_{n}\right)\right] o d o \\
\leq 32^{2 n} E \int_{S^{2}} o \dot{\mathfrak{o}}_{n} * \dot{\mathfrak{i}}_{n} o d o \\
\leq 32^{2 n} E\left|\dot{\mathfrak{i}}_{n}\right|^{2}
\end{aligned}
$$

is the general term of a convergent sum, and now the BorelCantelli lemma implies that the convergence of

5. 11

$$
\sum_{n \geq 0} \dot{\mathfrak{i}}_{n}=\sum_{n \geq 0} \mathfrak{h}_{n}+\sum_{n \geq 0} E\left(\dot{\mathfrak{i}}_{n}\right)=\sum_{n \geq 0} \mathfrak{h}_{n}+e(\mathfrak{i} t)
$$

is uniform on compacts.

$\mathrm{g}$ is therefore well-defined and continuous; that it solves 4.8 is clear; and that it lies in SO (3) follows from

5. 12

$$
\begin{aligned}
& g(t) * g(t) \\
& =\mathrm{g}(0)^{*} \mathrm{~g}(0)+\int_{0}^{t} \mathrm{~g}\left[d \mathrm{i}+* d \mathrm{j}+d \mathrm{j}^{*} d \mathrm{j}\right]^{*} \mathrm{~g} \\
& =\mathfrak{e} \quad t \geq 0
\end{aligned}
$$

and from the fact that $\operatorname{det}(g)(= \pm 1)$ is continuous and $=+1$ at $t=0$.

\section{CONVERGENCE PROOF}

Coming to the proof that $\lim _{n \uparrow \infty} \mathrm{g}_{n}=\mathrm{g}\left(=\sum_{n \geq 0} \dot{\mathrm{i}}_{n}\right)$, take $t \leq 1, j=\left[2^{n} t\right]$, 
and let us estimate

6.1

$$
\begin{aligned}
\mathrm{g}_{n}(t)-\mathrm{g}(0) & -\int_{0}^{t} \mathrm{~g}_{n}(s) \mathrm{j}(d s) \\
= & \mathrm{f}_{n}(t)-\int_{0}^{t} \mathrm{f}_{n}(s) \dot{\mathrm{j}}(d s) \\
& +\mathrm{g}_{n}\left(j 2^{-n}\right)-\mathrm{g}(0)-\sum_{i \leq j} \mathrm{~g}_{n}\left((i-1) 2^{-n}\right) \dot{\mathrm{j}}\left(\Delta_{i}\right),
\end{aligned}
$$

where

6.2

$$
\begin{aligned}
\mathfrak{f}_{n}(s) & =\mathfrak{g}_{n}(s)-\mathfrak{g}_{n}\left((i-1) 2^{-n}\right) & & s \in \Delta_{i} \\
\Delta_{i} & =\left[(i-1) 2^{-n}, i 2^{-n}\right) & & i \leq 2^{n} .
\end{aligned}
$$

P. Lévy's 4.3 implies that

$$
\max _{t \leq 1}\left|f_{n}(t)\right|<\sqrt{ } 32^{-n} \lg 2^{n} \quad n \uparrow \infty
$$

and that, up to terms of magnitude $2^{n}\left[\sqrt{ } 32^{-n} \lg 2^{n}\right]^{3}<2^{-n / 3}$,

6. 4

$$
\begin{aligned}
\mathfrak{g}_{n}\left(j 2^{-n}\right)-\mathfrak{g}(0)-\sum_{i \leq j} \mathfrak{g}_{n}\left((i-1) 2^{-n}\right) \mathrm{j}\left(\Delta_{i}\right) & \\
& =\sum_{i \leq j} \mathfrak{g}_{n}\left((i-1) 2^{-n}\right)\left(e\left[z\left(\Delta_{i}\right)\right]-\mathfrak{e}-\mathrm{j}\left(\Delta_{i}\right)\right) \\
& =\sum_{i \leq j} \mathfrak{g}_{n}\left((i-1) 2^{-n}\right) \mathfrak{w}_{i} \\
\mathfrak{w}_{i} & =\frac{1}{2}\left[\mathfrak{z}\left(\Delta_{i}\right)^{2}-\mathfrak{t} 2^{-n}\right] \quad i \leq 2^{n} .
\end{aligned}
$$

Itô's trick (5.6) and the semi-martingale method of 5.11 give 6.5

$$
\begin{aligned}
& P\left[\max _{j \leq 2^{n}}\left|\sum_{i \leq j} \mathfrak{g}_{n}\left((i-1) 2^{-n}\right) \mathfrak{w}_{i}\right| \geq 2^{-n / 3}\right] \\
& \quad \leq 2^{2 n / 3} E\left|\sum_{j \leq 2^{n}} \mathfrak{g}_{n}\left((i-1) 2^{-n}\right) \mathfrak{w}_{i}\right|^{2} \\
& \quad \leq 32^{2 n / 3} E \int_{S^{2}} o\left[\sum_{i \leq 2^{n}} \mathfrak{g}_{n} \mathfrak{w}_{i} * \sum_{i \leq 2^{n}} \mathfrak{g}_{n} \mathfrak{w}_{i}\right] o d o \\
& \quad=32^{2 n / 3} E \int_{S^{2}} o\left[\sum_{i \leq 2^{n}} \mathfrak{g}_{n} \mathfrak{w}_{i}^{*} \mathfrak{w}_{i}{ }^{*} \mathfrak{g}_{n}\right] o d o \\
& \quad=32^{2 n / 3} E \int_{S^{2}} o\left[\sum_{i \leq 2^{n}} \mathfrak{g}_{n}\left(\mathfrak{i}_{2} 2^{-2 n}+\mathfrak{i}_{3} 2^{-3 n}+\mathfrak{i}_{4} 2^{-4 n}\right) * \mathfrak{g}_{n}\right] o d o \\
& \leq 3\left(\left|\mathfrak{i}_{2}\right|+\left|\dot{\mathfrak{t}}_{3}\right|+\left|\dot{\mathfrak{t}}_{4}\right|\right) 2^{-n / 3}
\end{aligned}
$$

with constant $\dot{\mathfrak{i}}_{2}, \dot{\mathfrak{i}}_{3}, \dot{\mathfrak{i}}_{4}$, so that, thanks to the Borel-Cantelli lemma,
6. 6

$$
\left|\mathfrak{g}_{n}\left(j 2^{-n}\right)-\mathrm{g}^{\prime}(0)-\sum_{i \leq j} \mathrm{~g}_{n}\left((i-1) 2^{-n}\right) \mathrm{j}\left(\Delta_{i}\right)\right|<2^{-n / 3}
$$$$
n \uparrow \infty \text {. }
$$ 
As to $\int_{0}^{t} \mathrm{f}_{n} d \dot{\mathrm{j}}$, if $\varepsilon_{n}=\varepsilon_{n}(t)$ is the indicator of the sphere $\left|\mathrm{f}_{n}(t)\right|$ $<2^{-n / 3}$, then, as is clear from 6.3 ,

6. 7

$$
\int_{0}^{t} \mathrm{f}_{n} d \dot{\mathrm{j}}=\int_{0}^{t} \mathrm{f}_{n} \varepsilon_{n} d \dot{\mathrm{j}} \quad t \leq 1, n \uparrow \infty,
$$

and now

6.8

$$
\int_{0}^{t} \mathrm{f}_{n} d \mathrm{j}=\int_{0}^{t} \mathrm{f}_{n} \mathfrak{t} d s+\int_{0}^{t} \mathrm{f}_{n} \mathfrak{y}(d s)
$$

in conjunction with

6. 9

$$
\left|\int_{0}^{t} \mathfrak{f}_{n} \mathfrak{i} d s\right| \leq|\mathfrak{i}| 2^{-n / 3} \quad t \leq 1, n \uparrow \infty,
$$

6. 10

$$
\begin{aligned}
& P\left[\max _{t \leq 1}\left|\int_{0}^{t} f_{n} \varepsilon_{n} \mathfrak{y}(d s)\right| \geq 2^{-n / 4}\right] \\
& \leq 32^{n / 2} E \int_{S^{2}} o\left[\int_{0}^{1} f_{n} \varepsilon_{n} \mathfrak{h}(d s)^{*} \int_{0}^{1} f_{n} \varepsilon_{n} \mathfrak{y}(d s)\right] o d o \\
& \left.=-32^{n / 2} E \int_{S^{2}} o \int_{0}^{1} \varepsilon_{n} f_{n} \mathfrak{t} * f_{n} d s\right] o d o \\
& \leq 32^{n / 2}|\mathfrak{t}| 2^{-2 n / 3}=3|\mathfrak{t}| 2^{-n / 6},
\end{aligned}
$$

and the Borel-Cantelli lemma, justifies

$$
\max _{t \leq 1}\left|\int_{0}^{t} \mathrm{f}_{n} d \mathrm{j}\right|<2^{-n / 4} \quad n \uparrow \infty .
$$

Collecting all this, if $\mathfrak{o}_{n}=\mathrm{g}_{n}-\mathfrak{g}(0)-\int_{0}^{t} \mathfrak{g}_{n} d \mathfrak{j}$, then

$$
\max _{t \leq 1}\left|\mathfrak{o}_{n}(t)\right|<3 \cdot 2^{-n / 4} \quad n \uparrow \infty,
$$

and using K. Itô's method of stochastic differential in conjunction with $d \mathrm{j}+* d \mathrm{j}+d \mathrm{j} * d \mathrm{i}=0$ to check

6.13

$$
\mathrm{g}_{n} * \mathrm{~g}=\mathfrak{o}_{n} * \mathrm{~g}+e-\int_{0}^{t} \mathfrak{o}_{n}^{*} d \mathrm{j}^{*} \mathrm{~g}
$$

and estimating $\int_{0}^{t} \mathfrak{o}_{n} * d \mathrm{j} * \mathrm{~g}$ as we estimated $\int_{0}^{t} \mathrm{f}_{n} d \mathrm{j}$ justifies

6. 14

$$
\max _{t \leq 1}\left|\mathfrak{g}-\mathfrak{g}_{n}\right|=\max _{t \leq 1}\left|\mathfrak{g}_{n}{ }^{*} \mathfrak{g}-e\right|<2^{-n / 5} \quad n \uparrow \infty,
$$

completing the proof.

\section{COMPUTING THE GENERATOR}

$\mathfrak{g}_{\infty}=\mathfrak{g}=\sum_{n \geq 0} \dot{\mathfrak{l}}_{n}$ is a Brownian motion on $\mathrm{SO}(3)$ : in fact, it is con- 
tinuous and its Markovian and (left) group-invariant character is clear from the product integral

7. 1

$$
\mathfrak{g}_{\infty}\left(t_{2}\right)=\mathfrak{g}_{\infty}\left(t_{1}\right) \bigcap_{t_{1} \leq t<t_{2}} e[z(d s)] \quad t_{2} \geq t_{1} .
$$

Coming to its generator, we adapt the stochastic differential of K. Itô [1951; 59-65] to the present setting and find that for $f \in C^{2}[\mathrm{SO}(3)]$, 7.2

$$
\begin{aligned}
& f(\mathfrak{g}(t)) \\
& \quad=f(\mathfrak{g}(0))+\int_{0}^{t}[z(d s) \cdot g r a d] f(\mathfrak{g}) \\
& \quad+\int_{0}^{t} \frac{1}{2}[z(d s) \cdot \operatorname{grad}]^{2} f(\mathfrak{g}) \\
& \quad=f\left(g^{\prime}(0)\right)+\int_{0}^{t}\left[\mathfrak{l}^{1 / 2} x(d s) \cdot \operatorname{grad}\right] \mathfrak{f}(\mathfrak{g}) \\
& \quad+\int_{0}^{t}(\mathbb{S} f)(\mathfrak{g}) d s,
\end{aligned}
$$

where grad is short for $\left(\mathfrak{F}_{1},\left(\mathfrak{F}_{2},\left(\mathfrak{F}_{3}\right)\right.\right.$.

But now it is clear that

$$
\begin{aligned}
& \lim _{t \downarrow 0} t^{-1} E[f(\mathfrak{g}(t))-f(\mathfrak{h})] \\
= & \lim _{t \downarrow 0} t^{-1} E \int_{0}^{t}((\mathbb{S} f)(\mathfrak{g}) d s] \\
= & (\mathbb{S} f)(\mathfrak{h}) \quad \mathfrak{h}=\mathfrak{g}(0),
\end{aligned}
$$

and this completes the identification of $\mathfrak{g}_{\infty}=\mathfrak{g}=\sum_{n \geq 0} \dot{\mathrm{I}}_{n}$ as the SO (3) BROWNian motion with generator (S).

\section{ROLLING WITHOUT SLIPPING}

Consider the standard Brownian motion on the plane $R^{2} \times 0 \subset R^{3}$ with generator $\frac{1}{2}\left(\frac{\partial^{2}}{\partial x_{1}^{2}}+\frac{\partial^{2}}{\partial x_{2}^{2}}\right)$ and sample path $w: t \rightarrow x(t)=\left(x_{1}(t)\right.$, $x_{2}(t), 0$ ) and let a sphere of diameter 2 roll without slipping on the plane $R^{2} \times-1 \subset R^{3}$ while its center traces out the polygonal line joining the points $x\left(j 2^{-n}\right): j \geq 0$ of the plane $R^{2} \times 0$.

Concentrating on times $t \leq 1$, select $m=m(w)$ such that

8. 1

$$
\left|x\left(t_{2}\right)-x\left(t_{1}\right)\right|<\left(t_{2}-t_{1}\right)^{1 / 3} \quad 0 \leq t_{1} \leq t_{2} \leq 1, t_{2}-t_{1}<2^{-m}
$$

and $2^{-m / 3}<\lg 2$, so that, for $|\alpha| \leq 2^{-m / 3}, e\left[\alpha_{1} \mathrm{e}_{1}+\alpha_{2} \mathrm{e}_{2}+\alpha_{3} \mathrm{e}_{3}\right]$ is the 
counterclockwise rotation of angle $|\alpha|$ about the axis $\alpha$.

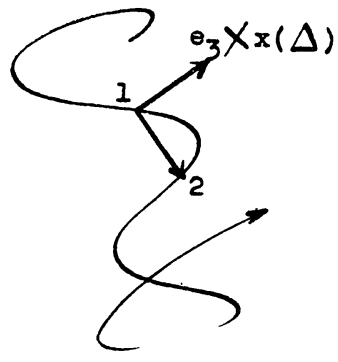

Given $n \geq m$ and $i \leq 2^{n}$, it is clear from the DIAGRAM that as $t$ grows from $t_{1}=$ $(i-1) 2^{-n}$ to $t_{2} \leq i 2^{-n}$ the sphere suffers a rotation

8.2 $e\left[-x_{2}(\Delta) \mathfrak{e}_{1}+x_{1}(\Delta) \mathfrak{e}_{2}\right] \quad \Delta=\left[t_{1}, t_{2}\right]$

of angle $|x(\Delta)|<2^{-n / 3} \quad$ counterclockwise about the axis $e_{3}$ cross $x(\Delta)$.

One sees at once that the total rotation up to time $t$ is identical in law to the $g_{n}(t)$ of 4 computed for

$$
\mathfrak{l}=\left(\begin{array}{lll}
1 & 0 & 0 \\
0 & 1 & 0 \\
0 & 0 & 0
\end{array}\right), \quad m_{1}=m_{2}=m_{3}=0, \quad \mathfrak{g}(0)=\mathfrak{e},
$$

and one concludes that $\lim _{n \uparrow \infty} g_{n}=g_{\infty}$ is the $\mathrm{SO}$ (3) Brownian motion with generator $\mathbb{S}=\frac{1}{2}\left(\mathfrak{S}_{1}^{2}+\mathbb{F}_{2}^{2}\right)$, permitting the identification of that motion with the total rotation up to time $t$ of a sphere of diameter 2 rolling without slipping on the plane $R^{2} \times-1$ as its center performs a standard Brownian motion on the plane $R^{2} \times 0$. C. D. Gorman [1958] also got a proof that $\lim _{n \uparrow \infty} g_{n}$ exists in the present case.

Consider, now, the path $\mathrm{g}_{\infty} e_{3}$ of the north pole $e_{3}=(0,0,1)$ : this motion is Markov; its generator $\mathscr{G}_{3}$ is $\frac{1}{2}\left(\mathfrak{(}_{1}^{2}+\left(\mathfrak{F}_{2}^{2}\right)\right.$ cut down to the coset space $\mathrm{SO}(3) / \mathrm{SO}(2)=\mathrm{S}^{2}$ :

8.4

$$
\mathscr{B}_{3}=\frac{1}{2}\left(\frac{1}{\sin \psi} \partial \bar{\psi} \sin \psi \frac{\partial}{\partial \psi}+\cot ^{2} \psi \frac{\partial^{2}}{\partial \phi^{2}}\right),
$$

where $\psi$ is colatitude and $\phi$ is longitude on $S^{2}$ (see 2.10).

$\mathbb{S}_{3}$ splits into the Legendre operator $(2 \sin \psi)^{-1} \frac{\partial}{\partial \psi} \sin \psi \frac{\partial}{\partial \psi}$ plus $\frac{1}{2} \cot ^{2} \psi \frac{\partial^{2}}{\partial \phi^{2}}$, and this splitting is reflected in the sample path ; in fact, colat $\left(\mathrm{g}_{\infty} e_{3}\right)$ is the process attached to the Legendre operator on $[0, \pi]$ and longitude $\left(\mathrm{g}_{\infty} e_{3}\right)$ is a standard circular Brownian motion independent of colat $\left(\mathfrak{g}_{\infty} e_{3}\right)$ run with the clock 


$$
\int_{0}^{t} \cot ^{2}\left[\operatorname{colat}\left(\mathrm{g}_{\infty} e_{3}\right)\right] d s .
$$

\section{REFERENCES}

J. L. Doob, Stochastic Processes. J. Wiley and Sons. New York 1953.

I. Gelfand and Z. Ya. Šapiro, Representations of the group of rotations in 3-dimensional space and their applications. Uspehi Mat. Nauk 7 (1952), 3-117; Amer. Math. Soc. Translations 2 (1956), 207-316.

C. D. Gorman, Brownian motion of rotation. Notices Amer. Math. Soc. 5 (1958) 196, abstract $553-22$.

K. Itô, Brownian motions in a Lie group. Proc. Japan Acad. 26 (1950), 4-10.

On a formula concerning stochastic differentials. Nagoya Math. J. 3 (1951), 55-65.

P. Lévy, Théorie de l'addition des variables aléatoires. Gauthier-Villars. Paris 1937. Processus stochastiques et mouvement brownien. Gauthier-Villars. Paris 1948.

W. Maak, Fastperiodische Funktionen. Springer Verlag. Berlin 1950.

F. Perrin, Étude mathématique du mouvement brownien de rotation. Ann. École Norm. Sup. 45 (1928), 1-51.

K. Yosida, Brownian motion in a homogeneous Riemannian space. Pacific J. 2 (1952), 263-96. 